\title{
Surface Plasmon Polariton Waves Propagation at the Boundary of Graphene Based Metamaterial and Corrugated Metal in THz range
}

\author{
Thanos IOANNIDIS ${ }^{1}$, TATJANA GRIC ${ }^{1,2,3}$, EdIK RAFAILOV ${ }^{2,4}$ \\ ${ }^{1}$ Department of Electronic Systems, Vilnius Gediminas Technical University, Vilnius, Lithuania \\ ${ }^{2}$ Aston Institute of Photonic Technologies, Aston University, Birmingham B4 7ET, UK \\ ${ }^{3}$ Semiconductor Physics Institute, Center for Physical Sciences and Technology, Vilnius, Lithuania \\ ${ }^{4}$ Interdisciplinary Center of Critical Technologies in Medicine, Saratov State University, 83 \\ Astrakhanskaya Street, Saratov 410012, Russia \\ *tatjana.gric@vgtu.lt
}

\begin{abstract}
Herein we study theoretically surface plasmon polariton (SPP) wave propagation along the nanostructured graphene-based metamaterial/corrugated metal interface. We apply the effective medium approximation formalism aiming to physically model nanostructured metamaterial. The transfer matrix approach is applied to compute the dispersion relationship for SPP waves. It has been concluded that the groove width $(a)$ and the chemical potential $(\mu)$ parameters have a dramatical impact aiming to engineer resonance surface plasmon frequencies of the propagation modes. Moreover, one can tune the bandgap corresponding to nonpropagation regime by modifying groove width parameter. The impact of the groove width $(a)$ and the chemical potential $(\mu)$ on the propagation length was investigated. The present work may have potential applications in optical sensing in terahertz frequency range.
\end{abstract}

(C) 2019 Optical Society of America under the terms of the OSA Open Access Publishing Agreement

\section{Introduction}

The fundamental optical excitations that are confined to a metal/dielectric interface are the Surface Plasmon Polaritons (SPPs), as described by Ritchie [1]. SPPs can be referred to as electromagnetic excitations existing at an interface between two media, of which at least one is conducting [2]. Investigations of spoof-plasmons in a semiconductor is becoming an increasingly active area of research. As a matter of fact, the presence of surface waves was originally demonstrated either for dielectric materials placed over a metal surface or for the metallic surface with a periodic repetition of obstacles of holes in the direction of propagation [3-5]. However, these surface waves can also be excited replacing the metallic surface with the semiconductor one [6]. Spoof plasmons are bound electromagnetic (EM) waves at frequencies outside the plasmonic range mimicking ("spoofing") surface plasmon polaritons (SPPs), which propagate on periodically corrugated metal surfaces [7].

Recently, an idea of engineering surface plasmons at lower frequencies was suggested. It was concluded in [8] that the existence of holes in the structure can lower the frequency of existing surface plasmons. Thus, by cutting holes or grooves in metal surfaces it is possible to take concepts such as highly localized waveguiding $[9,10]$ and superfocusing $[11,12]$ to lower frequencies, particularly to the $\mathrm{THz}$ regime [13], where plasmonics could enable near-field imaging and biosensing [14] with unprecedented sensitivity.

In recent years, electromagnetic waves propagating at an interface between a metal and a dielectric have been of significant interest. An experimental study of propagation of a $\mathrm{THz}$ Zenneck surface wave on an aluminium sheet is presented in [15]. The properties of long-range SPPs are reviewed in [16]. It is shown in [17] that a simple waveguide, namely a bare metal 
wire, can be used to transport terahertz pulses with virtually no dispersion, low attenuation, and with a remarkable structural simplicity.

The advent of the graphene has given rise to the unprecedented progress in actively tunable microwave, terahertz, and optical devices and structures during the last decade [18-20]. Especially, we have seen significant progress in $\mathrm{THz}$ generation and detection over the last decade which leads to the surging demand for $\mathrm{THz}$ wave devices. Single-layer graphene demonstrates a dynamically changeable conductivity, permittivity, and impedance [21] while being one-atom thick and efficiently tunable by varying the chemical potential. Up to now, a single-layer uniformand patterned graphene [22-26] being the simplest case for experimental studies has opened wide avenues for many applications. Though, a high degree of freedom for tunable devices is provided by double-layer graphene [27] and graphene-dielectric multilayers [18, 28-31]. Doing so, in contrast to noble metals, graphene plasmonic resonances can be dynamically tuned through electrostatic biasing and enable a new generation of reconfigurable plasmonic devices. Among the new perspectives opened with graphene-dielectric multilayer structures, hyperbolic metamaterials [29, 32-37], tunable beam steering [31], and Tamm surface plasmons [30] stand out. In addition, it is worthwhile noting, that surface-plasmon-polaritons (SPPs) at the interfaces of nanostructured metamaterials containing graphene [38], tunable surface waves at the interface separating different graphene-dielectric hyperbolic metamaterials [39], and tunable perfect absorption at mid-infrared frequencies [40] are of the particular importance for the scientific community. It is worthwhile noting that dispersion of graphenedielectric metamaterials can be engineered by changing the Fermi energy, e.g., see Refs. [32, 36].

Here, we propose the nanostructured graphene-based metamaterial/corrugated metal interface. We show that dispersion relation of SPPs can be adjusted by the shape of the textured grooves. Following the introduction, the remainder of this paper is structured as follows: Section 2 will describe the main building blocks comprising the system under consideration. In Section 3, the obtained results are discussed. Finally, in Section 4 conclusions are provided.

\section{Material properties and design}

The structure under consideration (Fig. 1) consists of two building blocks: grating made of $\mathrm{Si}$ $\left(\varepsilon=\varepsilon_{g}=12.25\right)$ and a slab of graphene-dielectric metamaterial. The function of the first building block is creation of higher-order transmission channel(s). In such a case, the coupling of the incident waves differs for the two opposite interfaces. The main its function is to attain tunability by changing the state from dielectric to ENZ, and then to plasmonic one, by engineering $\mu$ [28].

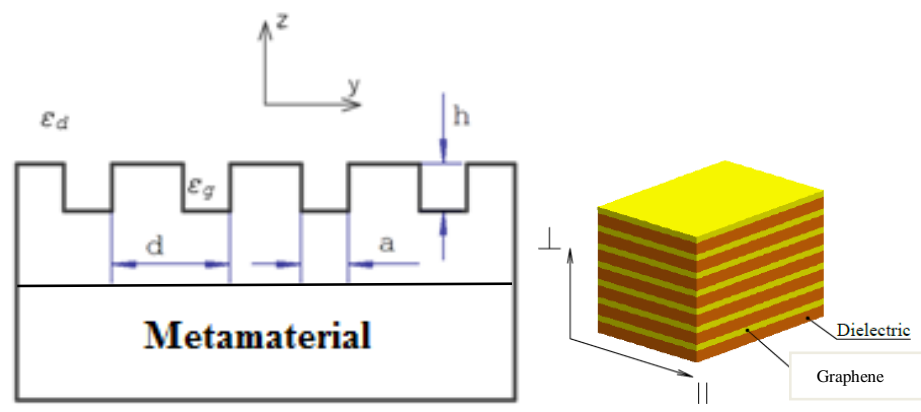

Fig. 1. Geometry of structured metamaterial surface

The effective-medium approach is applied aiming to describe the optical response of such a system. The former is justified if the wavelength of the radiation considered is much larger than the thickness of any layer. It is based on averaging the structure parameters. Hence, further in 
this paper we consider the effective homogeneous media for the semi-infinite periodic structures. The effective permittivities are as follows [41]:

$$
\begin{aligned}
& \varepsilon_{\mathrm{P}}^{m}=\frac{\varepsilon_{m g} d_{m g}+\varepsilon_{m d} d_{m d}}{d_{m g}+d_{m d}} \\
& \varepsilon_{\perp}^{m}=\frac{\varepsilon_{m g} \varepsilon_{m d}\left(d_{m g}+d_{m d}\right)}{\varepsilon_{m g} d_{m d}+\varepsilon_{m d} d_{m g}},
\end{aligned}
$$

where $\varepsilon_{m g}, \varepsilon_{m d}$ - are the permittivities of the graphene and dielectric layers correspondingly; $d_{m g}, d_{m d}$ - are the thicknesses of the graphene and dielectric layers correspondingly.

Matching the tangential components of the electrical and magnetic fields at the interface implies the dispersion relation for the surface modes localized at the boundary separating two anisotropic media [42]. We assume the permittivity $\varepsilon_{m g}(\omega)$ to be frequency dependent as the corresponding layer is represented by graphene.

Within the random-phase approximation and without an external magnetic field, graphene may be regarded as isotropic and the surface conductivity can be written as follows [21, 43], $\sigma=i e^{2} \mu / \pi h^{2}(\omega+i / \tau)$, where $\omega, h, e, \mu, \tau$ is the frequency, Planck constant, charge of an electron, chemical potential (Fermi energy), and phenomenological scattering rate, respectively. The Fermi energy $\mu$ can be straightforwardly obtained from the carrier density $n_{2 D}$ in a graphene sheet, $\mu=h v_{F} \sqrt{\pi n_{2 D}}, v_{F}$ is the Fermi velocity of electrons. It should be mentioned, that one can electrically control the carrier density $n_{2 D}$ by an applied gate voltage, thus leading to a voltage-controlled Fermi energy $\mu$. Here we assumed that the electronic band structure of a graphene sheet is unaffected by the neighboring layers. Thus, the effective permittivity $\varepsilon_{m g}$ of graphene can be calculated as follows [44]: $\varepsilon_{m g}=1+i \sigma / \varepsilon_{0} \omega d_{m g}$, where $\varepsilon_{0}$ is the permittivity in the vacuum. 

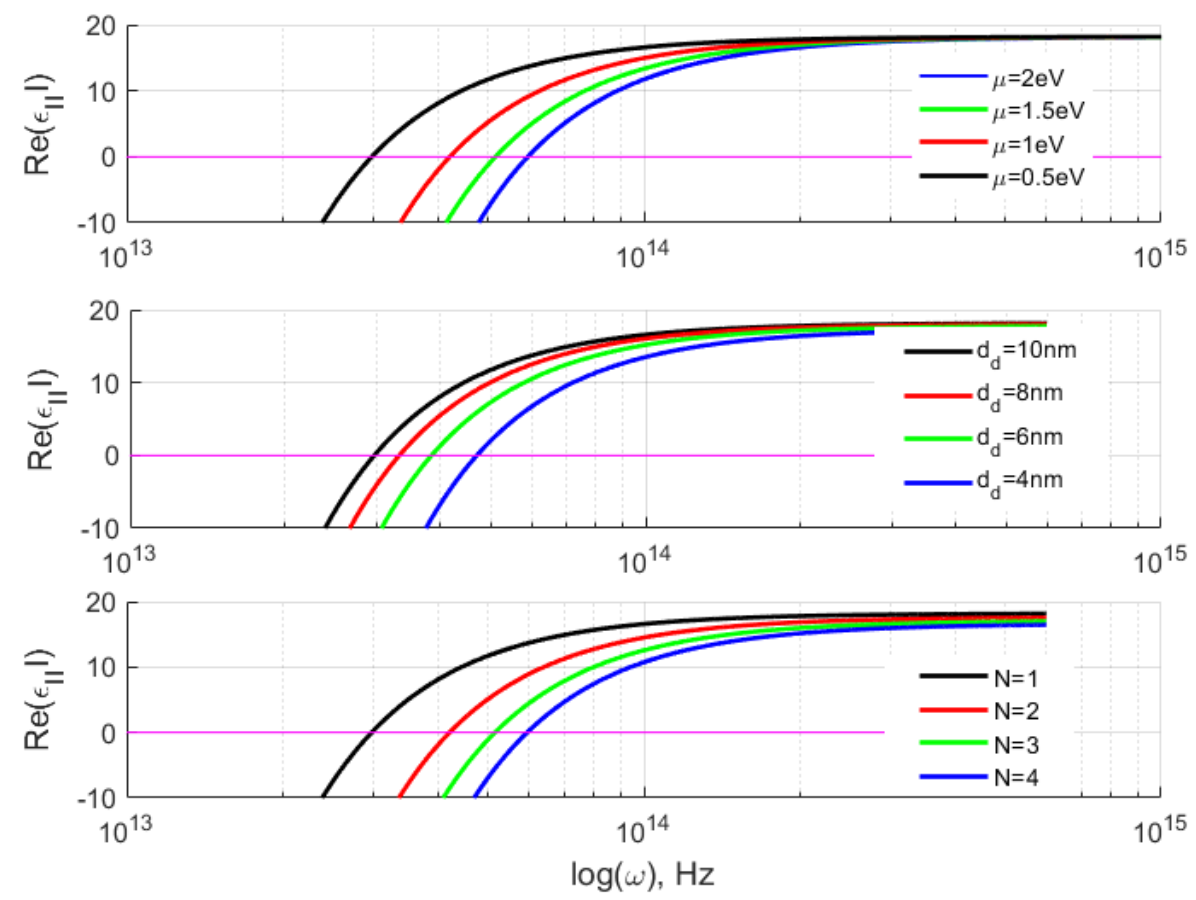

Fig. 2. The influence of (a), Fermi energy $\mu$, (b) thickness of dielectric $d_{m d}$, and (c) number of graphene sheets $N$ on the real part of $\varepsilon_{\|} . N=1, d_{m d}=10 \mathrm{~nm}$ in (a), $N=1, \mu=0.5 \mathrm{eV}$ in (b), and $d_{m d}=10, \mu=0.5 \mathrm{eV}$ in (c).

One can see that $\operatorname{Re}\left(\varepsilon_{\|}\right)$crosses zero at a frequency which depends on $\mu$. For instance, this happens at $30 \mathrm{THz}$ when $\mu=0.5 \mathrm{eV}$, and at $45 \mathrm{THz}$ when $\mu=1 \mathrm{eV}$. Changing $\mu$ may drastically shift the spectral range of transition from the effectively plasmonic to the effectively dielectric state. Note that the gate positioning will be considered at the next steps. Generally, electrical gating of a multilayer graphene-dielectric metamaterial is a challenging task. One of possible gating schemes is presented in Ref. [28]. In Ref. [45], chemical doping has been used while preparing each graphene layer using CVD, instead of electrical gating. In Ref. [46], the doublelayer graphene has been experimentally gated, and the method was presented by the authors as the one being usable for the structures composed of a larger number of graphene monolayers. So, from a practical point of view, this approach can be utilized also in the case of graphenedielectric metamaterial containing finite number of graphene layers.

In order to find the dielectric parameters of the effective medium describing gratings, consider a periodic assembly of parallel plates. The effective dielectric constants of such an assembly are as follows [47]

$$
\begin{aligned}
& \varepsilon_{x}=\varepsilon_{z}=\frac{(d-a) \varepsilon_{A g}+a \varepsilon_{g}}{d} \\
& \varepsilon_{y}=\frac{d}{(d-a) / \varepsilon_{A g}+a / \varepsilon_{g}}
\end{aligned}
$$

Here, $\varepsilon_{d}$ is the permittivity of the surrounding media, $\varepsilon_{A g}$ - is the permittivity of silver. 


\section{Results and discussion}

Herein, we present a simple example of polaritons in corrugated metal at $\mathrm{THz}$ frequencies. The wave vector $k$ [48] is plotted as a function of the frequency aiming to illustrate the properties of SPPs. We deal with Ag case [49]. It is assumed that the structure is surrounded by silicon, i. e. $\operatorname{Si}\left(\varepsilon=\varepsilon_{g}=12.25\right)$.

Figure 3(a) depicts the dispersion curves of SPPs at the boundary metamaterial/structured surface with $d=10 \mathrm{~nm}$. It is of particular importance to investigate the impact of the period on the dispersion curves of SPPs. Doing so, three different groove widths $a$ are considered. One may conclude from Figure 3, that the asymptotic frequency decreases with an increase in groove width. Figure 3(b) presents the losses of these SPPs as a function of frequency. It is worthwhile noting that the loss of SPPs is dramatically influenced by the increase in frequency. Besides that, it is of particular importance to note, that the forbidden region between the modes starts squeezing as presented in the Fig. 3(a) and the approach each other with the decrease of the groove width.

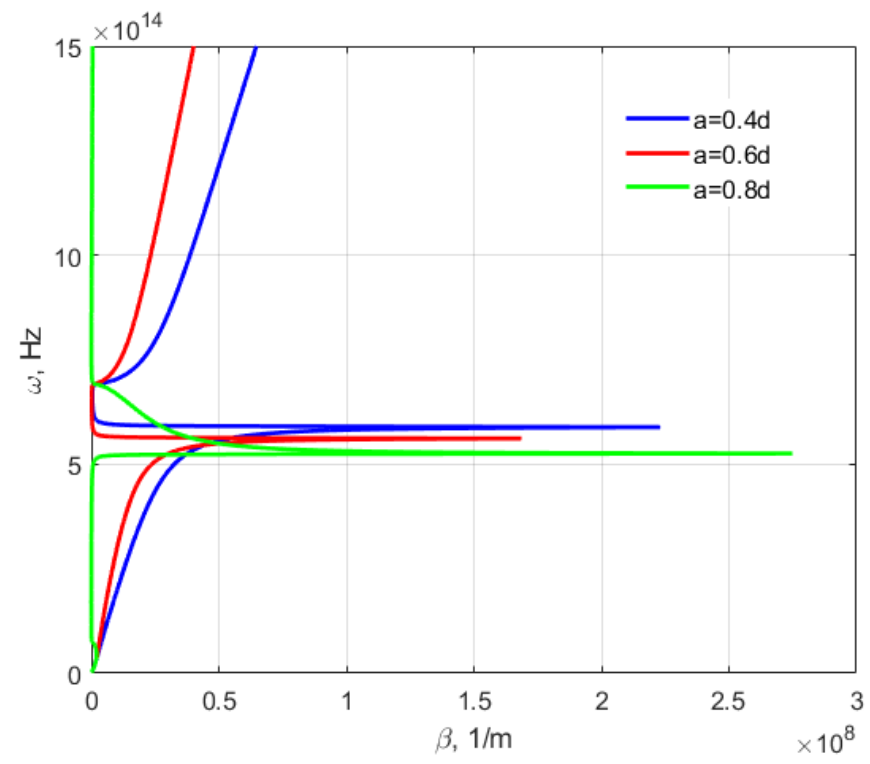

(a) 


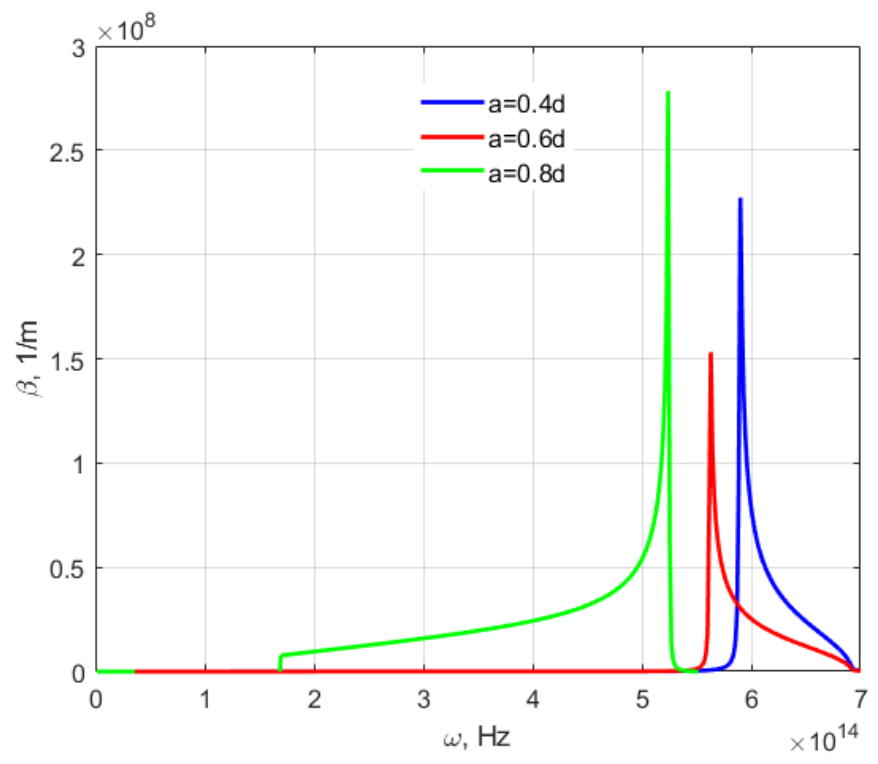

(b)

Fig. 3. (a) Dispersion curves for SPPs. (b) Attenuation coefficients of SPPs, lattice constant $d=10 \mathrm{~nm}$.

It is of particular interest to analyze the effect of the lattice constant $(d)$ on the dispersion of SPPs. The dispersion curves for SPPs at the boundary metamaterial/corrugated surface with different lattice constants $d=5,7,10 \mathrm{~nm}$, respectively are displayed in Figure 4(a). The groove parameter is $a=2 \mathrm{~nm}$ for all cases. Figure 4(b) shows the losses of SPPs for three cases. It can be concluded from Figure 4(b), that a larger loss of SPPs for a given frequency takes place in case of a smaller lattice constant. One might conclude from Figure 4(b), that losses of the SPPs are drastically influenced by the lattice constant. For a given frequency, an increase of the lattice constant may cause a significant reduction of the loss of spoof SPPs. A low-loss $\mathrm{THz}$ waveguiding system is essential because of the need for a compact, reliable, and flexible $\mathrm{THz}$ system for various applications. 


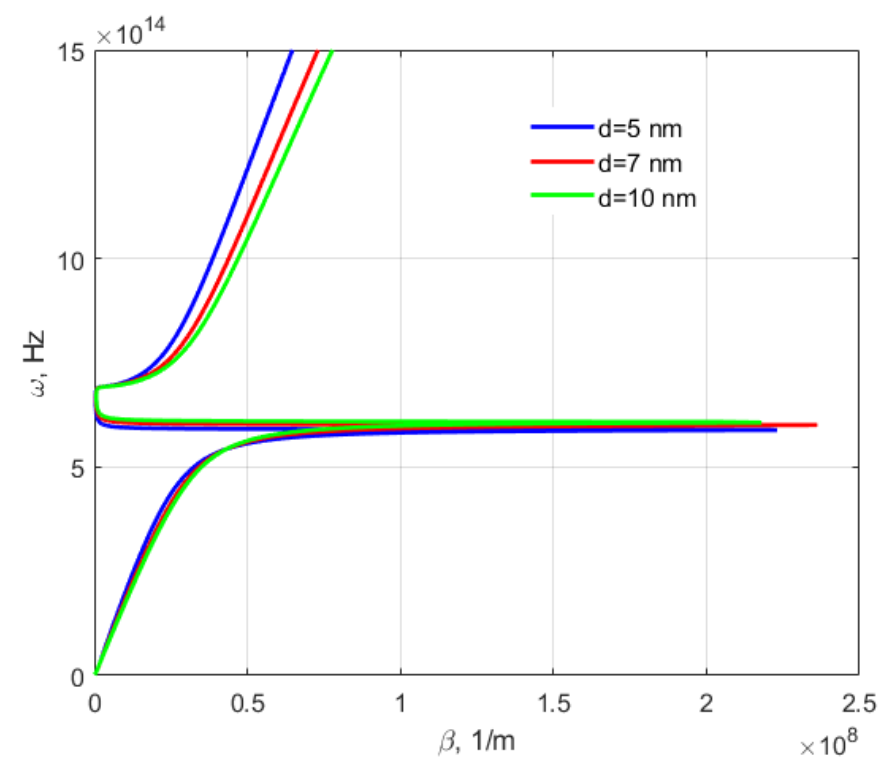

(a)

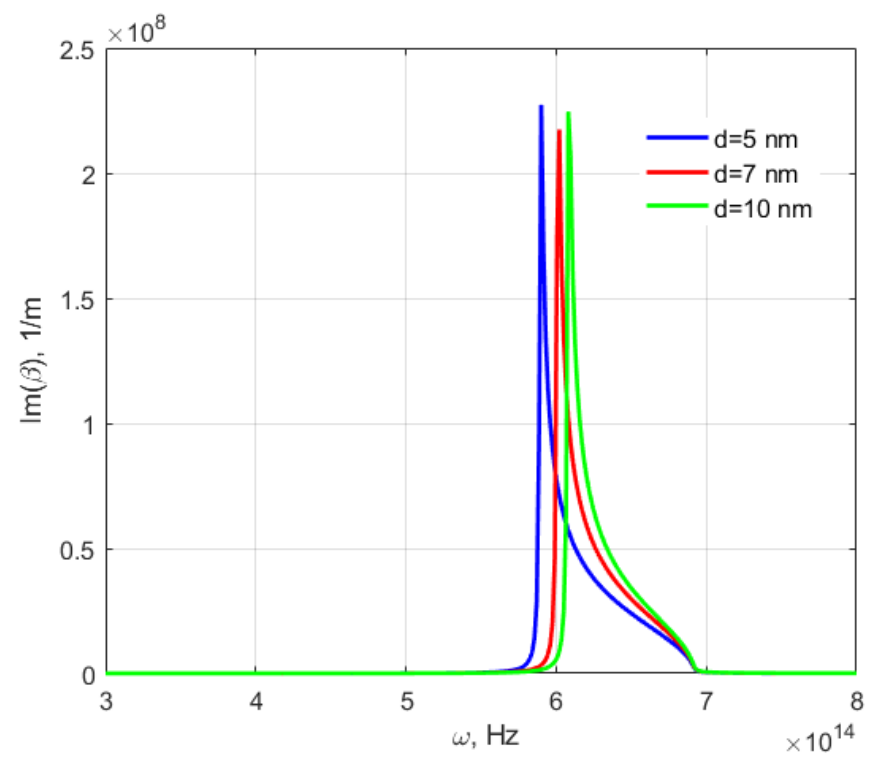

(b)

Fig. 4. Dispersion curves (a) and attenuation coefficients (b) of SPPs for different lattice constants $d=5,7,10 \mathrm{~nm}$, respectively. Parameters of grooves: $a=2 \mathrm{~nm}$.

The impact of the chemical potential on the propagating modes along metamaterial/corrugated metal interface is depicted in Figure 5. One may engineer the chemical potential $\mu$ of graphene by tuning the gate voltage or doping [44]. It is worthwhile noting, that the chemical potential has different values in these dispersion curves, i. e. $\mu=0.1 \mathrm{eV}, \mu=0.5 \mathrm{eV}, \mu=1 \mathrm{eV}, \mu=1.5 \mathrm{eV}$. It has been confirmed that the chemical potential has a dramatic impact modulating the modes. It should be noted that the resonance frequency for the modes increases with the increase of chemical potential. 


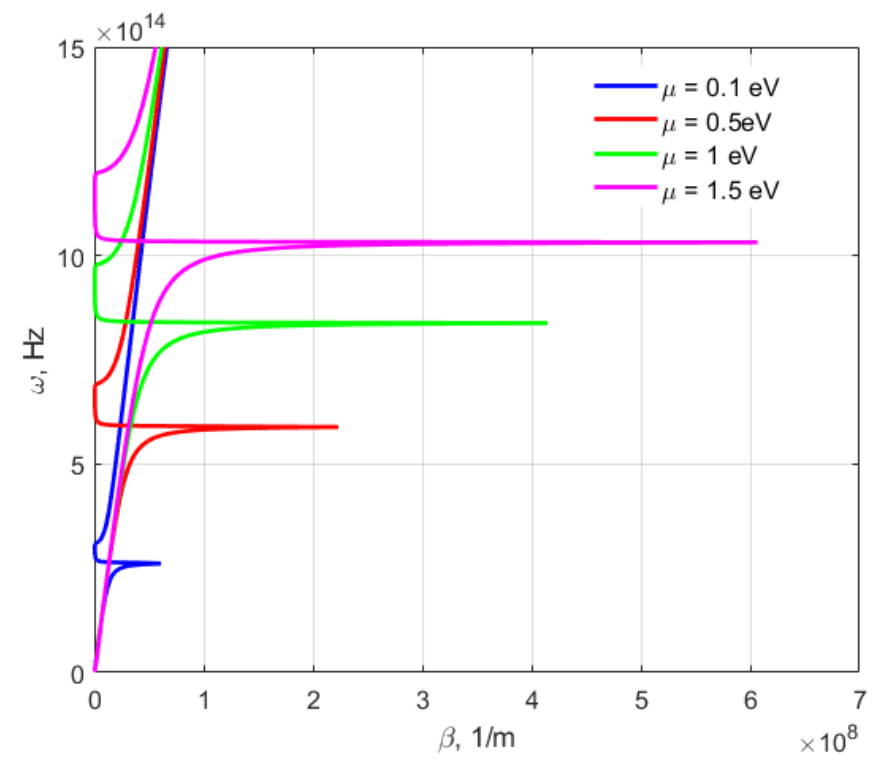

(a)

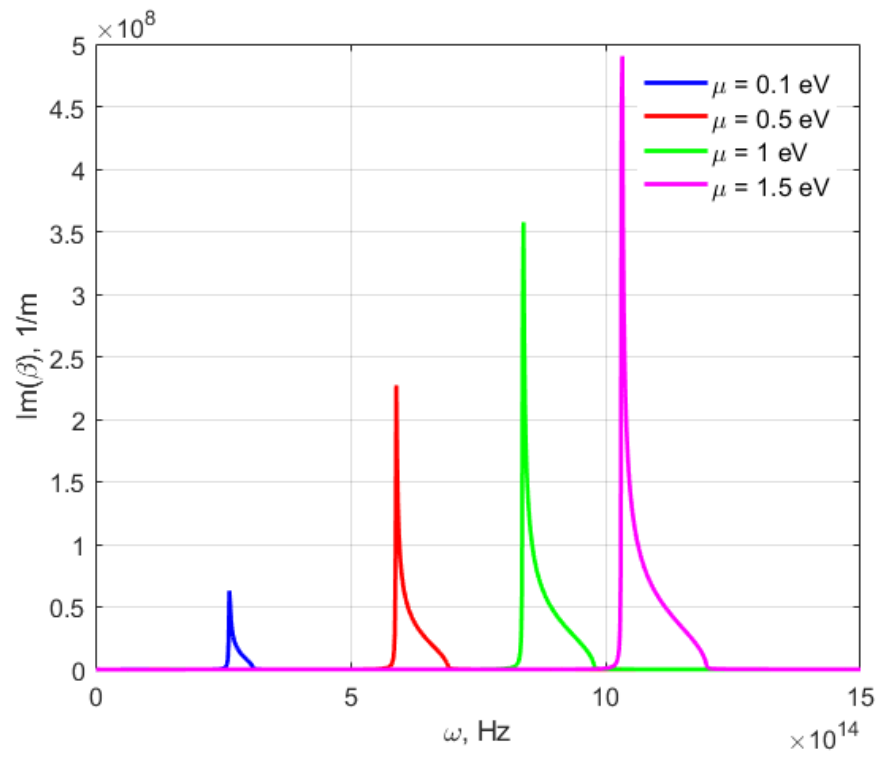

(b)

Fig. 5. Influence of chemical potential on the dispersion curves (a) and attenuation coefficients (b) of SPPs at nanostructure metamaterial interface with $d=10 \mathrm{~nm}, a=0.4 d$. 


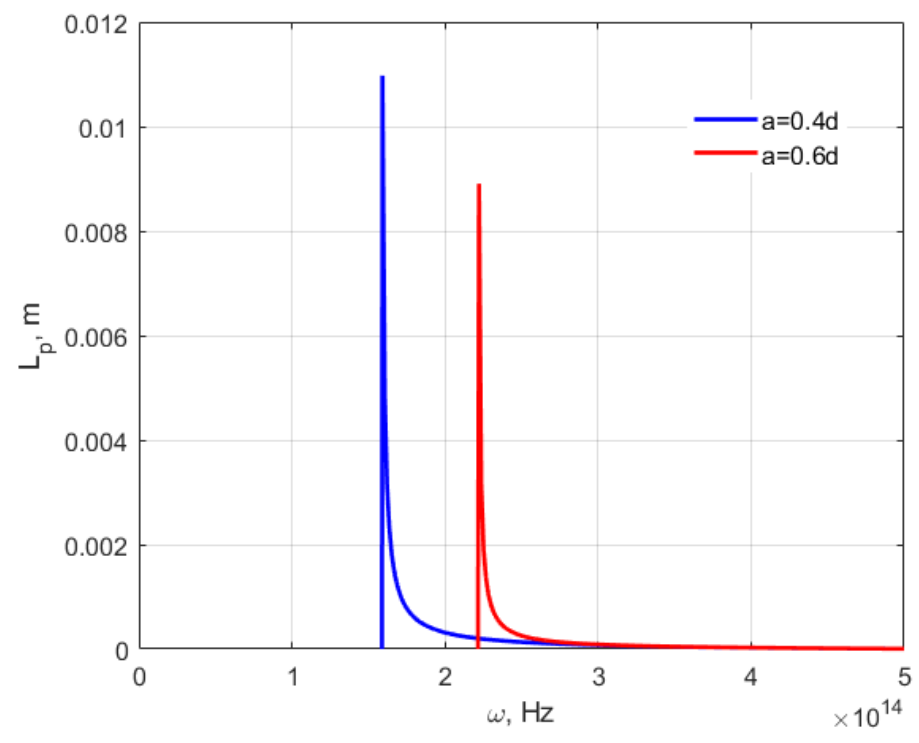

(a)

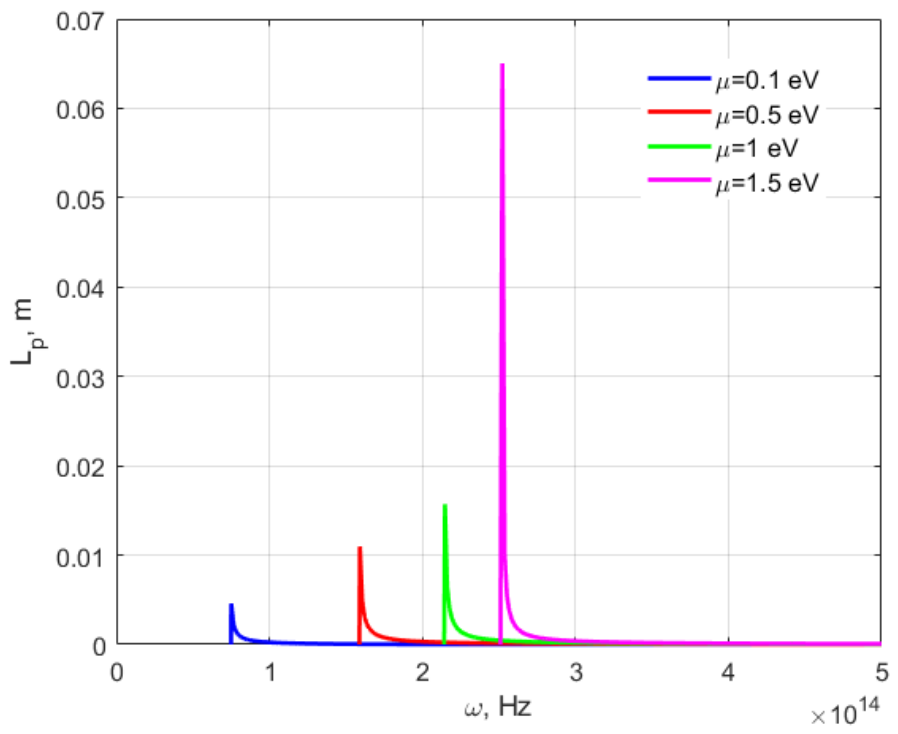

(b)

Fig. 6. Influence of groove width (a) and chemical potential (b) on the propagation length of surface wave modes as function of incidence frequency.

Fig. 6 displays the propagation length $L_{p}=\frac{1}{2 \operatorname{Im}(\beta)}$ as a function of the incidence terahertz frequency $(\omega=0.1-500 \mathrm{THz})$ for different values of groove width $(a=0.4 d ; a=0.6 d$ ) and chemical potential $(\mu=0.1 \mathrm{eV}, 0.5 \mathrm{eV}, 1 \mathrm{eV}, 1.5 \mathrm{eV})$. It is obvious that groove width $(a)$ and the chemical potential $(\mu)$ play a significant role in modulation of the propagation length of the modes. The propagation length of the modes increases increasing groove width and chemical 
potential. It is of particular interest to mention that the propagation length profiles of the modes follow the exponential decay. The usage of graphene allows for the increase the propagation length of the modes compared to ordinary interface modes.

\section{Conclusion}

The nanostructured metamaterial interface is used to excite the SPP waves. The dispersion relationship is computed by implementing the effective medium theory and transfer matrix approach and the following conclusions can be drawn:

- Surface wave modes propagate along the studied interface.

- One may tune the bandgap corresponding to non-propagation regime by engineering the groove width or chemical potential of graphene.

- The propagation length as a function of the terahertz frequency range is studied. It has been concluded that under appropriate parameters the propagation length can be modulated. The present method of surface wave modulation is quite simple in comparison with the corrugated structures [50].

- The proposed geometry can be used for optical sensing and wave propagation in the terahertz regime.

Herein, we have considered a formalism to analyze SPPs at the metamaterial/corrugated metal interface. In comparison with the previous works, our approach enables us to investigate the metamaterial case since it takes into account the permittivity of the metamaterial expressed by the effective medium theory. We have analyzed the properties of the dispersion and loss of SPPs at the metamaterial/corrugated metal boundary at the $\mathrm{THz}$ frequency range. The groove width drastically affects the asymptotic frequency of SPPs. On the other hand, the loss of SPPs is sensitive to all parameters of the surface structure. However, the performance of low-loss propagation for spoof SPPs can be achieved by an optimum design of the surface structure.

\section{Acknowledgement}

This project has received funding from the European Union's Horizon 2020 research and innovation programme under the Marie Sklodowska Curie grant agreement No 713694 and from Engineering and Physical Sciences Research Council (EPSRC) (Grant No. EP/R024898/1). E.U.R. also acknowledges support and the Russian Science Foundation (Grant No. 18-15-00172).

\section{References}

1. R. H. Ritchie, "Plasma losses by fast electrons in thin films," Phys. Rev. 106, 874 (1957).

2. H. Raether, Surface plasmons on smooth and rough surfaces and on gratings. Springer-Verlag: Berlin; 1988.

3. J. B. Pendry, L. Martin-Moreno, F. J. Garcia-Vidal, "Mimicking surface plasmons with structured surfaces," Science 305, 847-848 (2004)

4. F. J. Garcia-Vidal, L. Martin-Moreno, J. B. Pendry, "Surfaces with holes in them: New plasmonic metamaterials," J. Opt. A-Pure Appl. Op. 7, S97 (2005).

5. T. Jiang, L. Shen, X. Zhang, L.-X. Ran, "High-order modes of spoof surface Plasmon polaritons on periodically corrugated metal surfaces," Pr. Electromagn. Res. M. 8, 91-102 (2009).

6. T. Gric, M. S. Wartak, M. Cada, J. J. Wood, O. Hess, J. Pistora, "Spoof plasmons in corrugated semiconductors," J. Electromagnet. Wave. 29, 1899-1907 (2015).

7. A. Rusina, M. Durach, M. I. Stockman, "Theory of spoof plasmons in real metals," Appl. Phys. A 100(2), 375378 (2010).

8. S. I. Bozhevolnyi, V. S. Volkov, E. Devaux, T. W. Ebbesen, "Channel plasmon-polariton guiding by subwavelength metal grooves," Phys. Rev. Lett. 95, 046802 (2005).

9. B. Lamprecht, J. R. Krenn, G. Schider, H. Ditlbacher, M. Salerno, N. Felidj, A. Leitner, F. R. Aussenegg, J. C. Weeber, "Surface plasmon propagation in microscale metal stripes," Appl. Phys. Lett. 79, 51 (2001).

10. S. A. Maier, P. G. Kik, H. A. Atwater, S. Meltzer, E. Harel, B. E. Koel, A. A. G. Requicha, "Local detection of electromagnetic energy transport below the diffraction limit in metal nanoparticle plasmon waveguides," Nat. Mater. 2, $229-232$ (2003).

11. S. I. Bozhevolnyi, V. S. Volkov, E. Devaux, J.-Y. Laluet, T. W. Ebbesen, "Channel plasmon subwavelength waveguide components including interferometers and ring resonators," Nature 440, 508-511 (2006). 
12. A. V. Krasavin, N. I. Zheludev, "Active plasmonics: Controlling signals in Au/Ga waveguide using nanoscale structural transformations," Appl. Phys. Lett. 84, 1416-1418 (2004).

13. A. V. Krasavin, A. V. Zayats, N. I. Zheludev, “Active control of surface plasmon-polariton waves," J. Opt. A: Pure Appl. Opt. 7, S85 (2005).

14. P. Andrew, W. L. Barnes, "Energy transfer across a metal film mediated by surface plasmon polaritons," Science 306, 1002-1005 (2004).

15. T.-I. Jeon, D. Grischkowsky, "THz Zenneck surface wave (THz surface plasmon) propagation on a metal sheet,” Appl. Phys. Lett. 88, 061113 (2006).

16. P. Berini, "Long-range surface plasmon polaritons," Advances in Optics and Photonics 1, 484-588 (2009).

17. K. Wang, D. M. Mittleman, "Metal wires for terahertz wave guiding," Nature 432(18), 376-379 (2004).

18. A. N. Grigorenko, M. Polini, and K. S. Novoselov, "Graphene plasmonics," Nat. Photon. 6, 749-758 (2012).

19. A. K. Geim and K. S. Novoselov, "The rise of graphene," Nat. Mat. 6, 183-191 (2007).

20. F. Bonaccorso, Z. Sun, T. Hasan, and A. C. Ferrari, "Graphene Photonics and Optoelectronics," Nat. Photon. 4. 611-622 (2010).

21. L. A. Falkovsky, “Optical properties of graphene,” J. Phys.: Conf. Ser. 129(1), 012004 (2008).

22. Y. Yao, M. A. Kats, P. Gevenet, N. Yu, Y. Song, J. Kong, and F. Capasso, "Broad Electrical Tuning of Graphene-Loaded Plasmonic Antennas," Nano Lett. 13(3), 1257-1264 (2013).

23. A. Yu. Nikitin, F. Guinea, and L. Martin-Moreno, "Resonant plasmonic effects in periodic graphene antidot arrays," Appl. Phys. Lett. 101(15), 151119 (2012).

24. Z. Fang, Z. Liu, Y.Wang, P. M. Ajayan, P. Nordlander, and N. J. Halas, "Graphene-Antenna Sandwich Photodetector," Nano Lett. 12(7), 3808-3813 (2012).

25. H. Hajian, I. D. Rukhlenko, P. T. Leung, H. Caglayan, and E. Ozbay, "Guided plasmon modes of a graphenecoated Kerr slab," Plasmonics 11(3), 735-741 (2016).

26. A. N. Morozovska, A. I. Kurchak, and M. V. Strikha, "Graphene Exfoliation at a Ferroelectric Domain Wall Induced by the Piezoelectric Effect: Impact on the Conductance of the Graphene Channel," Phys. Rev. Appl. 8(5), 054004 (2017).

27. D. Rodrigo, A. Tittl, O. Limaj, F. J. Garcia de Abajo, V. Pruneri, and H. Altug, "Double-layer graphene for enhanced tunable infrared plasmonics," Light: Sci. Appl. 6, e16277 (2017).

28. I. Khromova, A. Andryieuski, and A. Lavrinenko, "Ultrasensitive terahertz/infrared waveguide modulators based on multilayer graphene metamaterials," Laser Photon. Rev. 8(6), 916-923 (2014).

29. I. Crassee, J. Levallois, A. L. Walter, M. Ostler, A. Bostwick, E. Rotenberg, T. Seyller, D. van der Marel, and A. B. Kuzmenko, "Giant Faraday rotation in single- and multilayer graphene," Nat. Phys. 7, 48-51 (2011).

30. H. Hajian, H. Caglayan, and E. Ozbay, "Long-range Tamm surface plasmons supported by graphene-dielectric metamaterials," J. Appl. Phys. 121(3), 033101 (2017).

31. B. Orazbayev, M. Beruete, and I. Khromova, "Tunable beam steering enabled by graphene metamaterials," Opt. Express 24(8), 8848-8861 (2016).

32. M. A. K. Othman, C. Guclu, and F. Capolino, "Graphene-dielectric composite metamaterials: evolution from elliptic to hyperbolic wavevector dispersion and the transverse epsilon-near-zero condition," J. Nanophoton. 7(1), 073089 (2013)

33. M. A. K. Othman, C. Guclu, and F. Cappolino, "Graphene-based tunable hyperbolic metamaterials and enhanced near-field absorption,” Opt. Express 21(6), 7614-7632 (2013).

34. B. Zhu, G. Ren, S. Zheng, Z. Lin, and S. Jian, "Nanoscale dielectric-graphene-dielectric tunable infrared waveguide with ultrahigh refractive indices," Opt. Express 21(14), 17089-17096 (2013).

35. Y. Xiang, J. Guo, X. Dai, S. Wen, and D. Tang, "Engineered surface Bloch waves in graphene-based hyperbolic metamaterials," Opt. Express 22(3), 3054-3062 (2014).

36. I. V. Iorsh, I. S. Mukhin, I. V. Shadrinov, P. A. Belov, and Y. S. Kivshar, "Hyperbolic metamaterials based on multilayer graphene structures,” Phys. Rev. B 87(7), 075416 (2013).

37. Y. Xiang, X. Dai, J. Guo, H. Zhang, S. Wen, and D. Tang, "Critical coupling with graphene-based hyperbolic metamaterials," Sci. Rep. 4, 5483 (2014)

38. T. Gric, "Surface-Plasmon-Polaritons at the Interface of Nanostructured Metamaterials," Prog. Electromag. Res. M 46, 165-172 (2016)

39. T. Gric and O. Hess, "Tunable surface waves at the interface separating different graphene-dielectric composite hyperbolic metamaterials," Opt. Express 25(10), 11466-11476 (2017).

40. J. Wu, L. Jiang, J. Guo, X. Dai, Y. Xiang, and S. Wen, "Tunable perfect absorption at infrared frequencies by a graphene-hBN hyper crystal," Opt. Express 24(15), 17103-17114 (2016).

41. V. M. Agranovich and V. E. Kravtsov, "Notes on crystal optics of superlattices," Solid State Commun. 55(1), 85-90 (1985).

42. I. Iorsh, A. Orlov, P. Belov, and Y. Kivshar, "Interface modes in nanostructured metal-dielectric metamaterials," Appl. Phys. Lett., 99, 151914 (2011).

43. G.W. Hanson, "Dyadic Green's functions and guided surface waves for a surface conductivity model of graphene," J. Appl. Phys. 103(6), 064302 (2008).

44. A. Vakil, N. Engheta, "Transformation optics using graphene," Science 332(6035), 1291-1294 (2011).

45. Y.-C. Chang, C.-H. Liu, C.-H. Liu, S. Zhang, S. R. Marder, E. E. Narimanov, Z. Zhong, and T. B. Norris, "Realization of mid-infrared graphene hyperbolic metamaterials," Nat. Commun. 7, 10568 (2016). 
46. J. S. Gomez-Diaz, C. Moldovan, S. Capdevila, J. Romeu, L. S. Bernard, A. Magrez, A. M. Ionescu, and J. Perruisseau-Carrier, "Self-biased reconfigurable graphene stacks for terahertz plasmonics," Nat. Commun. 6, 6334 (2015)

47. M. Born, E. Wolf, Principles of Optics (Cambridge, 1999).

48. T. Gric, O. Hess, "Controlling hybrid-polarization surface plasmon polaritons in dielectric-transparent conducting oxides metamaterials via their effective properties," Journal of Applied Physics 122, 193105 (2017)

49. P. B. Johnson, R. W. Christy, "Optical constants of the noble metals," Phys. Rev. B 6, 4370 (1972).

50. R. S. Anwar, H. Ning, L. Mao, "Recent advancements in surface plasmon polaritons-plasmonics in subwavelength structures at microwave and terahertz regime," Digital Communications and Networks 4(4), 244-257 (2017). 